\title{
Strong divisibility and lcm-sequences
}

\author{
Andrzej Nowicki \\ Nicolaus Copernicus University, \\ Faculty of Mathematics and Computer Science, \\ 87-100 Torun, Poland, (e-mail: anow@mat.uni.torun.pl).
}

\begin{abstract}
Let $R$ be a gcd-domain (for example let $R$ be a unique factorization domain), and let $\left(a_{n}\right)_{n \geqslant 1}$ be a sequence of nonzero elements in $R$. We prove that $\operatorname{gcd}\left(a_{n}, a_{m}\right)=$ $a_{\operatorname{gcd}(n, m)}$ for all $n, m \geqslant 1$ if and only if

$$
a_{n}=\prod_{d \mid n} c_{d} \text { for } n \geqslant 1,
$$

where $c_{1}=a_{1}$ and $c_{n}=\operatorname{lcm}\left(a_{1}, a_{2}, \ldots, a_{n}\right) / \operatorname{lcm}\left(a_{1}, a_{2}, \ldots, a_{n-1}\right)$ for $n \geqslant 2$. All equalities with gcd and lcm are determined up to units of $R$.
\end{abstract}

\section{Introduction}

Let $R$ be a commutative unique factorization domain, and let $\left(a_{n}\right)_{n \geqslant 1}$ be a sequence of nonzero elements of $R$. Put $c_{1}=a_{1}$ and $c_{n}=\operatorname{lcm}\left(a_{1}, a_{2}, \ldots, a_{n}\right) / \operatorname{lcm}\left(a_{1}, a_{2}, \ldots, a_{n-1}\right)$ for $n \geqslant 2$. Then $\left(c_{n}\right)$ is a sequence of nonzero elements of $R$ and it is well-defined up to units. We will say that $\left(c_{n}\right)$ is the lcm-sequence of $\left(a_{n}\right)$. A sequence $\left(a_{n}\right)_{n \geqslant 1}$ of nonzero elements of $R$ is called a strong divisibility sequence if $a_{\operatorname{gcd}(m, n)}$ is a greatest common divisor of $a_{m}$ and $a_{n}$ for all $m, n \geqslant 1$.

Consider the case $R=\mathbb{Z}[x]$, and let $a_{n}=x^{n}-1$ for $n \geqslant 1$. The $n$th cyclotomic polynomial is commonly defined by the formula $\Phi_{n}(x)=\left(x-\omega_{1}\right) \cdots\left(x-\omega_{\varphi(n)}\right)$, where $\varphi$ is the Euler totient function and $\omega_{1}, \ldots, \omega_{\varphi(n)}$ are the primitive $n$th roots of unity. It is well known that for any $n$ we have the equality $a_{n}=\prod_{d \mid n} \Phi_{d}(x)$. Applying the Möbius inversion formula, we obtain the equality $\Phi_{n}(x)=\prod_{d \mid n} a_{n}^{\mu(n / d)}$, in which $\mu$ is the Möbius function. This formula may be used as a definition of $\Phi_{n}(x)$. Recently, Tomasz Ordowski presented a new alternative definition of $\Phi_{n}(x)$. He observed that $\left(\Phi_{n}(x)\right)$ is the lcm-sequence of $\left(a_{n}\right)$, that is, $\Phi_{1}(x)=x-1$ and

$$
\Phi_{n}(x)=\frac{\operatorname{lcm}\left(x^{1}-1, x^{2}-1, \ldots, x^{n}-1\right)}{\operatorname{lcm}\left(x^{1}-1, x^{2}-1, \ldots, x^{n-1}-1\right)} \quad \text { for } n \geqslant 2 .
$$

Tomasz Ordowski observed also that there exists a similar formula for integer values of $\Phi_{n}(x)$. If $b \geqslant 2$ is an integer, then $\Phi_{1}(b)=b-1$, and

$$
\Phi_{n}(b)=\frac{\operatorname{lcm}\left(b^{1}-1, b^{2}-1, \ldots, b^{n}-1\right)}{\operatorname{lcm}\left(b^{1}-1, b^{2}-1, \ldots, b^{n-1}-1\right)} \text { for } n \geqslant 2 .
$$


This means that the sequence $\left(\Phi_{n}(b)\right)_{n \geqslant 1}$ is equal to the lcm-sequence of $\left(b^{n}-1\right)_{n \geqslant 1}$.

It is well known that $\left(x^{n}-1\right)_{n \geqslant 1}$ and $\left(b^{n}-1\right)_{n \geqslant 1}$ are strong divisibility sequences in $\mathbb{Z}[x]$ and $\mathbb{Z}$, respectively. In this article we show that every strong divisibility sequence has the above properties.

In this article a sequence $\left(a_{n}\right)_{n \geqslant 1}$ of nonzero elements of $R$ is called special if there exists a sequence $\left(b_{n}\right)_{n \geqslant 1}$ of nonzero elements in $R$ such that $a_{n}=\prod_{d \mid n} b_{d}$ for any $n \geqslant 1$. Note that if a sequence $\left(a_{n}\right)$ is special, then its associated sequence $\left(b_{n}\right)$ is unique. The uniqueness follows from the Möbius inversion formula, applied to the field of fractions of $R$; we have $b_{n}=\prod_{d \mid n} a_{d}^{\mu(n / d)}$ for any $n \geqslant 1$.

It is known (Mathematical Olympiad, Iran 2001, Problem 5, see for example [1] p. 218) that every strong divisibility sequence in $\mathbb{Z}$ is special. Recently, Nathan Bliss, Ben Fulan, Stephen Lovett and Jeff Sommars proved in [3] that the same is true for strong divisibility sequences in arbitrary unique factorization domains. Thus, if $\left(a_{n}\right)$ is a strong divisibility sequence in a factorization domain $R$, then there exists a unique sequence $\left(b_{n}\right)$ of nonzero elements in $R$ with $b_{1}=a_{1}$ and $a_{n}=\prod_{d \mid n} b_{d}$. The authors of [3] proved also that there exist special sequences which are not strong divisibility sequences.

The main theorem of this article states that if $\left(a_{n}\right)_{n \geqslant 1}$ is a sequence of nonzero elements in $R$ and $\left(c_{n}\right)$ is the lcm-sequence of $\left(a_{n}\right)$, then $\left(a_{n}\right)$ is a strong divisibility sequence if and only if

$$
a_{n}=\prod_{d \mid n} c_{d} \text { for all } n \geqslant 1
$$

Applying the main theorem to the sequences $\left(x^{n}-1\right)$ and $\left(b^{n}-1\right)$, we obtain the above mentioned formulas of Ordowski. It is well-known that the Fibonacci sequence, the sequences of the form $\left(a^{n}-b^{n}\right)$ with $\operatorname{gcd}(a, b)=1$, and other known sequences have the strong divisibility property. Applying the main theorem and the Möbius inversion formula to these sequences, we obtain new formulas for some lcm-sequences and strong divisibility sequences.

We restricted our attention to an arbitrary unique factorization domain $R$. The main theorem of this article we prove for more general domains. We assume only that $R$ is a gcd-domain.

\section{Notations and preliminary facts}

Let $S$ be a domain, that is, $S$ is a commutative ring with identity without zero divisors. Let $a_{1}, \ldots, a_{n}$ be nonzero elements of $S$. A greatest common divisors (abbreviated as gcd) of $a_{1}, \ldots, a_{n}$ is a nonzero element $d$ in $S$ such that $d \mid a_{i}$ for $i=1, \ldots, n$, and if $0 \neq d^{\prime} \in S, d^{\prime} \mid a_{i}$ for $i=1, \ldots, n$, then $d^{\prime} \mid d$. A least common multiple (abbreviated as $\mathrm{lcm}$ ) of $a_{1}, \ldots, a_{n}$ is a nonzero element $m$ in $S$ such that $a_{i} \mid m$ for $i=1, \ldots, n$, and if $0 \neq m^{\prime} \in S, a_{i} \mid m^{\prime}$ for $i=1, \ldots, n$, then $m \mid m^{\prime}$.

Throughout this article we assume that $R$ is a gcd-domain, that is, $R$ is a domain and any two nonzero elements in $R$ have a greatest common divisor ([5]). It follows from the definition of gcd-domains, that if $n \geqslant 1$ and $a_{1}, \ldots, a_{n}$ are nonzero elements in $R$, then 
there exist a greatest common divisor and a least common multiple of $a_{1}, \ldots, a_{n}$. We adopt the notation $\left(a_{1}, \ldots, a_{n}\right)$ and $\left[a_{1}, \ldots, a_{n}\right]$ for the greatest common divisor and the least common multiple, respectively, of $a_{1}, \ldots, a_{n}$. Note that the elements $\left(a_{1}, \ldots, a_{n}\right)$ and $\left[a_{1}, \ldots, a_{n}\right]$ are determined only up to units. We can allow this ambiguity in our article.

Unique factorization domains, Bezout domains and valuation domains belong to the class of gcd-domains. Every gcd-domain is integrally closed. If $R$ is a gcd-domain, then the polynomial ring $R[x]$ is also a ged-domain ([5]).

We say that two nonzero elements $a, b \in R$ are relatively prime, if $(a, b)=1$. It should be carefully noted that we are not assuming that $(a, b)$ is a linear combination of $a$ and $b$. Let us note some properties of gcd and lcm.

Proposition 1.1. If $a, b, c$ are nonzero elements of a gcd-domain $R$, then:

(1) $((a, b), c)=(a,(b, c))=(a, b, c), \quad[[a, b], c]=[a,[b, c]]=[a, b, c]$;

(2) $(a c, b c)=(a, b) c, \quad[a c, b c]=[a, b] c$;

(3) $(a, b)[a, b]=a b$;

(4) if $d=(a, b)$, then $(a / d, b / d)=1$;

(5) if $(a, b)=1$ and $(a, c)=1$, then $(a, b c)=1$;

(6) if $(a, b c)=1$, then $(a, b)=1$ and $(a, c)=1$;

(7) if $(a, b)=1$ and $a \mid b c$, then $a \mid c$;

(8) if $(a, b)=1, a \mid c$ and $b \mid c$, then $a b \mid c$.

Recall that the equalities which appear in this proposition are determined up to units. The proof of this proposition is elementary, so we omit it.

Let $\left(a_{n}\right)_{n \geqslant 1}$ be a sequence of nonzero elements of a gcd-domain $R$. Recall that $\left(a_{n}\right)$ is called a strong divisibility sequence if $\left(a_{m}, a_{n}\right)=a_{(m, n)}$ for all $m, n \geqslant 1$. The following two theorem from [3] will play an important role. They are stated in [3] for unique factorization domains, but they are also valid (with the same proofs) for arbitrary ged-domains.

Theorem 1.2 ([3]). If $\left(a_{n}\right)_{n \geqslant 1}$ is a strong divisibility sequence of a gcd-domain $R$, then there exists a unique sequence $\left(b_{n}\right)_{n \geqslant 1}$ of nonzero elements in $R$ such that $a_{n}=\prod_{d \mid n} b_{d}$ for all $n \geqslant 1$.

Theorem $1.3([3])$. Let $R$ be a gcd-domain and let $\left(b_{n}\right)_{n \geqslant 1}$ be a sequence of nonzero elements of $R$. Let $\left(a_{n}\right)_{n \geqslant 1}$ be the sequence such that $a_{n}=\prod_{d \mid n} b_{d}$. The sequence $\left(a_{n}\right)$ is a strong divisibility sequence if and only if, for all positive integers $m$ and $n$ such that $m \nmid n$ and $n \nmid m$, the elements $b_{m}$ and $b_{n}$ are relatively prime.

Assume again that $\left(a_{n}\right)_{n \geqslant 1}$ is a sequence of nonzero elements of a gcd-domain $R$. Put

$$
e_{1}=1 \text { and } e_{n+1}=\left[e_{n}, a_{n}\right] \text { for } n \geqslant 1 .
$$

Then $e_{n+1}=\left[a_{1}, \ldots, a_{n}\right]$ and $e_{n} \mid e_{n+1}$ for all $n \geqslant 1$. Denote by $c_{n}$ the element $e_{n+1} / e_{n}$. Then $\left(c_{n}\right)_{n \geqslant 1}$ is a sequence of nonzero elements in $R$. We call it the lcm-sequence of $\left(a_{n}\right)$. Note, that $c_{1}=a_{1}$ and

$$
c_{n}=\frac{\left[a_{1}, \ldots, a_{n}\right]}{\left[a_{1}, \ldots, a_{n-1}\right]} \text { for all } n \geqslant 2
$$


If for example $\left(a_{n}\right)$ is a constant sequence, $a_{n}=a$ for all $n \geqslant 1$ with $0 \neq a \in R$, then $c_{1}=a$ and $c_{n}=1$ for all $n \geqslant 2$. The lcm-sequence of the geometric sequence $a_{n}=q^{n}$ with $0 \neq q \in R$ is equal to the constant sequence $c_{n}=q$. If $R=\mathbb{Z}$ and $a_{n}=n$ !, then $c_{n}=n$. The lcm-sequence of the sequence $a_{n}=n$ is of the form $\left(c_{n}\right)$, where

$$
c_{n}= \begin{cases}p, & \text { if } n=p^{s} \text { for some prime } p \text { and integer } s \geqslant 1 \\ 1, & \text { otherwise }\end{cases}
$$

The first few terms of the lcm-sequence of the triangular numbers $t_{n}=n(n+1) / 2$ are: $1,3,2,5,1,7,2,3,1,11,1,13,1,1,2,17,1,19$.

\section{The main theorem}

Theorem 2.1. Let $\left(a_{n}\right)_{n \geqslant 1}$ is a sequence of nonzero elements in a gcd-domain $R$, and let $\left(c_{n}\right)_{n \geqslant 1}$ be the lcm-sequence of $\left(a_{n}\right)$. Then the following two conditions are equivalent.

(1) $\left(a_{n}\right)$ is a strong divisibility sequence.

(2) $a_{n}=\prod_{d \mid n} c_{d}$ for all $n \geqslant 1$.

Proof. $\quad(1) \Rightarrow(2)$. Assume that $\left(a_{n}\right)$ is a strong divisibility sequence. Then, by Theorem [1.2, there exists a unique sequence $\left(b_{n}\right)_{n \geqslant 1}$ of nonzero elements in $R$, such that $a_{n}=\prod_{d \mid n} b_{d}$ for all $n \geqslant 1$. Consider the sequence $\left(e_{n}\right)_{n \geqslant 1}$ defined by $e_{1}=1$ and $e_{n+1}=\left[e_{n}, a_{n}\right]$ for $n \geqslant 1$. We will prove, using an induction with respect to $n$, that for every $n \geqslant 1$ we have the equality

$$
e_{n+1}=b_{1} b_{2} \cdots b_{n}
$$

For $n=1$ it is obvious, because $e_{2}=\left[e_{1}, a_{1}\right]=a_{1}=b_{1}$. Now let $n \geqslant 2$, and assume that $e_{n}=b_{1} \cdots b_{n-1}$. Then we have

$$
e_{n+1}=\left[e_{n}, a_{n}\right]=\left[\prod_{k=1}^{n-1} b_{k}, \prod_{d \mid n} b_{d}\right]=\left[A \cdot B, A \cdot b_{n}\right],
$$

where $A$ is the product of the elements $b_{d}$ with $d<n$ and $d \mid n$, and $B$ is the product of all elements of the form $b_{d}$ with $d<n$ and $d \nmid n$. Since $\left(a_{n}\right)$ is a strong divisibility sequence, we know by Theorem 1.3 and Proposition 1.1 that the elements $B$ and $b_{n}$ are relatively prime. Hence, $\left[B, b_{n}\right]=B b_{n}$ and so, we have:

$$
e_{n+1}=\left[A B, A b_{n}\right]=A\left[B, b_{n}\right]=A B b_{n}=\left(\prod_{k=1}^{n-1} b_{k}\right) \cdot b_{n}=b_{1} \cdots b_{n} .
$$

Thus, by induction, we have a proof of $(*)$. But, by the definition of the lcm-sequence of $\left(a_{n}\right), c_{1}=a_{1}$ and for $n \geqslant 2$ we have $c_{n}=e_{n+1} / e_{n}=\left(b_{1} \cdots b_{n}\right) /\left(b_{1} \cdots b_{n-1}\right)=b_{n}$. Whence $b_{n}=c_{n}$ for all $n \geqslant 1$. Therefore, $a_{n}=\prod_{d \mid n} b_{d}=\prod_{d \mid n} c_{d}$ for $n \geqslant 1$. 
$(2) \Rightarrow(1)$. Assume now that $a_{n}=\prod_{d \mid n} c_{d}$ for $n \geqslant 1$, and let $m \geqslant 1$ be a fixed integer. Denote by $U$ the product of the elements $c_{d}$ with $d<m$ and $d \mid m$, and denote by $V$ the product of all elements of the form $c_{d}$ with $d<m$ and $d \nmid m$. Then we have:

$$
U V c_{m}=\prod_{i=1}^{m} c_{i}=e_{m+1}=\left[e_{m}, a_{m}\right]=\left[\prod_{i=1}^{m-1} c_{i}, \prod_{d \mid m} c_{d}\right]=\left[U V, U c_{m}\right]=U\left[V, c_{m}\right] .
$$

Hence, $\left[V, c_{m}\right]=V c_{m}$ and hence, the elements $V$ and $c_{m}$ are relatively prime. Recall that $V$ is the product of all elements $c_{d}$ with $d<m$ and $d \nmid m$. This implies (by Proposition 1.1) that if $d<m$ and $d \nmid m$, then the elements $c_{d}$ and $c_{m}$ are relatively prime.

We proved that if $n, m \geqslant 1, n \mid m$ and $m \nmid n$, then the elements $c_{n}$ and $c_{m}$ are relatively prime. Therefore, by Theorem 1.3, the sequence $\left(a_{n}\right)$ is a strong divisibility sequence.

It is well-known that the sequence $a_{n}=x^{n}-1$ is a strong divisibility sequence in the polynomial ring $\mathbb{Z}[x]$. In this case we have the equalities $x^{n}-1=\prod_{d \mid n} \Phi_{d}(x)$, where each $\Phi_{n}(x)$ is the $n$th cyclotomic polynomial. We know also, by Theorem 2.1, that $x^{n}-1=\prod_{d \mid n} c_{d}$, where $\left(c_{n}\right)_{n \geqslant 1}$ is the lcm-sequence of $\left(a_{n}\right)$. Applying the Möbius inversion formula we obtain that $\Phi_{n}(x)=c_{n}$ for all $n \geqslant 1$, and hence, we have:

Corollary 2.2 (Ordowski). Let $a_{n}=x^{n}-1$. Then $\Phi_{1}(x)=a_{1}$ and

$$
\Phi_{n}(x)=\frac{\left[a_{1}, a_{2}, \ldots, a_{n-1}, a_{n}\right]}{\left[a_{1}, a_{2}, \ldots, a_{n-1}\right]} \text { for } n \geqslant 2 .
$$

By the same way we obtain similar formulas for integer values of cyclotomic polynomials. Let $b \geqslant 2$ be an integer and let $a_{n}=b^{n}-1$ for $n \geqslant 1$. Then $\left(a_{n}\right)_{n \geqslant 1}$ is a strong divisible sequence in $\mathbb{Z}$ and $b^{n}-1=\prod_{d \mid n} \Phi_{d}(b)$ for $n \geqslant 1$. Applying Theorem 2.1 and the Möbius inversion formula we again obtain that $\left(\Phi_{n}(b)\right)_{n \geqslant 1}$ is the lcm-sequence of $\left(a_{n}\right)$. Therefore we have the following corollary.

Corollary 2.3 (Ordowski). Let $b \geqslant 2$ be an integer and let $a_{n}=b^{n}-1$ for $n \geqslant 1$. Then

$$
\Phi_{n}(b)=\frac{\left[a_{1}, a_{2}, \ldots, a_{n-1}, a_{n}\right]}{\left[a_{1}, a_{2}, \ldots, a_{n-1}\right]} \text { for } n \geqslant 2 .
$$

In particular, $\left[M_{1}, \ldots, M_{n}\right] /\left[M_{1}, \ldots, M_{n-1}\right]=\Phi_{n}(2)$, where each $M_{n}$ is the Mersenne number $2^{n}-1$.

It is easy to prove and it is well-known that the sequence $a_{n}=x^{n}-y^{n}$ is a strong divisibility sequence in the polynomial ring $\mathbb{Z}[x, y]$. In this case we have the equalities $x^{n}-y^{n}=\prod_{d \mid n} \Psi_{n}(x, y)$, where $\Psi_{n}(x, y)$ are polynomials in $\mathbb{Z}[x, y]$ defined by

$$
\Psi_{n}(x, y)=y^{\varphi(n)} \Phi_{n}(x / y)=\prod_{d \mid n}\left(x^{d}-y^{d}\right)^{\mu(n / d)}
$$


for all $n \geqslant 1$ (see for example [8]). Thus, $\left[a_{1}, \ldots, a_{n}\right] /\left[a_{1}, \ldots, a_{n-1}\right]=\Psi_{n}(x, y)$ for all $n \geqslant 2$. Similarly, if $u>v \geqslant 1$ are relatively prime integers, then the sequence $a_{n}=u^{n}-v^{n}$ is a strong divisible sequence in $\mathbb{Z}$, and again we have the equalities $\left[a_{1}, \ldots, a_{n}\right] /\left[a_{1}, \ldots, a_{n-1}\right]=\Psi_{n}(u, v)$ for all $n \geqslant 2$.

Examples and properties of strong divisibility sequences can be found in many books and articles (see for example: [6], [10], [3], 7], 9] 150-152). For each such sequence we have, by Theorem 2.1, an associated formula connected with its lcm-sequence. Consider several examples. The sequence of repunits,

$$
u_{n}=\underbrace{11 \ldots 1}_{n}=\frac{10^{n}-1}{9},
$$

is a strong divisible sequence in $\mathbb{Z}$. The first few terms of the lcm-sequence are:

$1, u_{2}, u_{3}, 101, u_{5}, 91, u_{7}, 10001,1001001,9091, u_{11}, 9901, u_{13}, 909091,90090991$.

The sequence of the Fibonacci numbers, $F_{1}=F_{2}=1, F_{n+2}=F_{n+1}+F_{n}$, is a strong divisibility sequence in $\mathbb{Z}([1])$. The first few terms of its lcm-sequence are:

$1,1,2,3,5,4,13,7,17,11,89,6,233,29,61,47,1597,19,4181,41,421,199$.

Let $\left(F_{n}(x)\right)_{n \geqslant 1}$ be the sequence of Fibonacci polynomials, that is,

$$
F_{1}(x)=1, F_{2}(x)=x \text { and } F_{n+2}(x)=x F_{n+1}(x)+F_{n}(x) \text { for } n \geqslant 1 .
$$

It is a strong divisibility sequence in $\mathbb{Z}[x]([4])$. The Chebyshev polynomials of the second kind $([2])$, which satisfy $U_{0}(x)=1, U_{1}(x)=2 x, U_{n+2}(x)=2 x U_{n+1}-U_{n}(x)$, also form a strong divisibility sequence in $\mathbb{Z}[x]([4])$. The same holds for the 3 -variable polynomials $S_{n}(x, y, z)$ which satisfy $S_{1}=1, S_{2}=x, S_{n}=x S_{n-1}+y S_{n-2}$ for $n$ even and $S_{n}=$ $z S_{n-1}+y S_{n-2}$ for $n$ odd ([4]. Also, other examples of strong divisibility polynomial sequences are constructed and discussed in [3].

Let us end this article with the following corollary, which is an immediately consequence of Theorem 2.1 and Theorem 3 in [3].

Corollary 2.4. Let $\left(a_{n}\right)_{n \geqslant 1}$ be a strong divisibility sequence in a gcd-domain $R$. If $n \geqslant 2$ has prime factorization $n=p_{1}^{\alpha_{1}} \cdots p_{s}^{\alpha_{s}}$, then

$$
\frac{\left[a_{1}, \ldots, a_{n}\right]}{\left[a_{1}, \ldots, a_{n-1}\right]}=\frac{a_{n}}{\left[a_{n / p_{1}}, a_{n / p_{1}}, \ldots, a_{n / p_{s}}\right]} .
$$

Acknowledgment. The author thanks Mr. Tomasz Ordowski for his interesting formulas. 


\section{References}

[1] T. Andrescu, Z. Feng, G. Lee Jr., Mathematical Olympiads 2000-2001. Problems and Solutions from Around the World, Mathematical Association of America, 2003.

[2] E. J. Barbeau, Pell's equation, Problem Books in Mathematics. Springer-Verlag, New York, 2003.

[3] N. Bliss, B. Fulan, S. Lovett and J. Sommars, Strong divisibility, cyclotomic polynomials, and iterated polynomials, American Mathematical Monthly, 120(2013), 519-536.

[4] V. E. Hoggatt, M. Bicknell-Johnson, Divisibility properties of polynomials in Pascal's triangle, Fibonacci Quart. 16 (1978), no. 6, 501513.

[5] I. Kaplansky, Commutative Rings, Chicago, London 1974.

[6] C. Kimberling, Strong divisibility sequences with nonzero initial term, Fibonacci Quart. 16 (6)(1978), 541544.

[7] M. Majewski, A. Nowicki, From Generalized Binomial Symbols to $\beta$ - and $\alpha$-sequences, PNG Journal of Mathematics Computing \& Education, 4(1998), 73-78.

[8] K. Motose, On values of cyclotomic polynomials VII, Bull. Fac. Sci. Tech. Hirosaki Univ. 7(2004) 1-8.

[9] A. Nowicki, Silnie i Symbole Newtona, Podróże po Imperium Liczb 11, Olsztyn, Toruń, 2013.

[10] A. Schinzel, Second order strong divisibility sequences in an algebraic number field, Archivum Mathematicum (Brno), 23(1987), 181-186.

[11] N. N. Vorobiev, Fibonacci Numbers, Basel: Birkhauser Verlag, 1992. 